\title{
RADIATION EFFECT ON MHD BLOOD FLOW THROUGH A TAPERED POROUS STENOSED ARTERY WITH THERMAL AND MASS DIFFUSION
}

\author{
M. SHARMA and R.K. GAUR \\ Department of Bioscience, CASH, Mody University of Science and Technology \\ Lakshmangarh, Rajasthan, INDIA \\ E-mail:madhusharma5dec@gmail.com \\ B.K. SHARMA* \\ Department of Mathematics, Birla Institute of Technology and Science \\ Pilani, Rajasthan, INDIA \\ E-mail: bhupen_1402@yahoo.co.in
}

\begin{abstract}
A mathematical model for MHD blood flow through a stenosed artery with Soret and Dufour effects in the presence of thermal radiation has been studied. A uniform magnetic field is applied perpendicular to the porous surface. The governing non-linear partial differential equations have been transformed into linear partial differential equations, which are solved numerically by applying the explicit finite difference method. The numerical results are presented graphically in the form of velocity, temperature and concentration profiles. The effects of various parameters such as the Reynolds number, Hartmann number, radiation parameter, Schmidt number and Prandtl number, Soret and Dufour parameter on the velocity, temperature and concentration have been examined with the help of graphs. The present results have an important bearing on the therapeutic procedure of hyperthermia, particularly in understanding/regulating blood flow and heat transfer in capillaries.
\end{abstract}

Key words: Soret and Dufour effects, MHD, blood flow, stenosis, chemical reaction.

\section{Introduction}

The study of blood flow through arteries is of considerable importance in many cardiovascular diseases. The poor circulation of blood in our body due to blockage in arteries is a major cause of health risks. Arteries carry oxygenated blood with nutrients from the heart to each cell of the body, in the circulatory system of the human body. Blood is a thick red liquid circulating in the blood vessels. It has a strong nourishing effect on the human body and serves as one of the basic substances constituting the human body. Blood is a marvelous fluid which is an important factor of life. For the last few decades, the theoretical and experimental studies of blood flow through the circulatory system of living mammals, has been the subject of scientific research. A huge amount of literature is now available on the subject, e.g., [1-3], but little attention has been paid to the study of heat and mass transfer effects on blood flow through tapered arteries. Characteristics of blood flow through an artery in the presence of multi-stenosis were studied by Chakravarty and Sannigrahi [4] The investigation of basic BFD flow problems attracts interest due to the numerous proposed applications in bioengineering and medical sciences.

The study of bio-fluids under the presence of a magnetic field with dissipation finds its applications in various upcoming fields like innovative drug targeting, surgical operations, etc. Haik et al. [5] reported a $30 \%$ decrease in blood flow rate when subjected to a high magnetic field of $10 \mathrm{~T}$ while Yadav et al. [6] showed a similar reduction in blood flow rate but at a much smaller magnetic field of $0.002 \mathrm{~T}$. Sharma et al. [7] presented a mathematical model for the hydro-magnetic bio-fluid flow in the porous medium with Joule

\footnotetext{
* To whom correspondence should be addressed
} 
effect. A theoretical analysis of blood flow and heat transfer in a permeable vessel in the presence of an external magnetic field was made by Sinha et al. [8]. Shit and Roy [9] studied the effects of induced magnetic field on blood flow through a constricted channel, and demonstrated that increasing the values of the magnetic field reduces the velocity of the blood flow at the center. Rahbari et al. [10] made an analytical study on blood flow containing nanoparticles through porous blood vessels in the presence of the magnetic field using the Homotopy Perturbation Method (HPM). Blood flow in a large blood vessel has a profound influence on the efficiency of thermal therapy treatment. Electromagnetic heat, such as short waves and microwaves, sends heat up to 2 inches into the tissue and muscles. It works best for injuries in joints, muscles, and tendons. Moreover, hyperthermia treatment has been demonstrated as effective during cancer therapy in recent years. Its objective is to raise the temperature of pathological tissues above cytotoxic temperatures $\left(41-45^{\circ} \mathrm{C}\right)$ without overexposing healthy tissues [11]. Heat and mass transfer of blood flow considering its pulsatile hydro-magnetic rheological nature under the presence of viscous dissipation, Joule heating and a finite heat source was discussed by Sharma et al. [12]. Sinha and Shit [13] investigated the combined effects of thermal radiation and MHD heat transfer blood flow through a capillary. Thermal radiation effect on inclined arterial blood flow through a non-Darcian porous medium with magnetic field was discussed by Sharma et al. [14 ]. Recently, Tripathi and Sharma [15] discussed the heat and mass transfer in MHD two-phase blood flow with radiation.

There are many important technological problems that concern the flow of chemically-reacting fluid mixtures. Many biological fluid systems are examples of such mixtures. For example, blood is a complex mixture of plasma, proteins, cells, and a variety of other chemicals that is modeled usually in a homogenized sense as a single constituent fluid. Blood is maintained in a delicate balance by a variety of chemical reactions, some that aid its coagulation and others its dissolution. Gnaneswara Reddy [16] reported the impacts of chemical reaction and thermal radiation on hydromagnetic convective boundary layer slip flow. Sharma and Gaur [17] studied the effect of variable viscosity on chemically reacting magneto-blood flow with heat and mass transfer. Recently, MHD third order blood flow in an irregular channel though a porous medium with homogeneous/heterogeneous reactions was discussed by Gnaneswara Reddy [18].

When heat and mass transfer occur simultaneously in a moving fluid, the relations between the fluxes and the driving potentials are more intricate in nature. It is now known that the mass transfer caused by the temperature gradient is called the Soret effect, while the heat transfer caused by the concentration effect is called the Dufour effect. The Dufour effect was recently found to be of order of considerable magnitude so that it cannot be neglected (Eckert and Drake [19]). The Dufour and Soret effects on unsteady MHD mixed convection for a heat generating fluid with thermal radiation and chemical reaction was analysed by Sharma et al. [20, 21]. The Soret and Dufour effects on the magnetohydrodynamic (MHD) flow of the Casson fluid over a stretched surface were discussed by Hayat et al. [22]. Recently, Hayat et al. [23] and Sharma et al. [24] analysed the Soret and Dufour effects on MHD Jeffrey fluid flow in a curved channel and straight artery.

The main motivation for any mathematical analysis of physiological fluid flows is to have a better understanding of the particular flow being modeled. If there is a similarity between the results obtained from the analysis and experimental and clinical data, then the mechanism of flow can at least be explained. An accurate mathematical study can help explain the major contributing factors to many flows in the human body because peristalsis is evident in many physiological flows. When comparing results the mathematical model and the experimental data, it is desirable that the data obtained from experimental research be as close as possible to the actual physiological parameter being analyzed. In spite of all these studies, the Dufour and Soret effects on MHD blood flow through stenosed artery with thermal radiation and chemical reaction has received little attention. Hence, the main object of the present investigation is to study the importance of the Soret and Dufour effect in the presence of thermal radiation in blood flow. A Newtonian model of blood flow is taken for this study. Chemical reaction is also studied for the blood flow in the presence of mild stenosis in porous tapered artery 


\section{Problem formulation}

Let $(r, \theta, z)$ be the cylindrical polar coordinate system with $r=0$ as the axis of symmetry of the tube. Consider the flow of an incompressible Newtonian fluid of constant viscosity $\mu$ and density $\rho$ in a tube having length $L$, and take $u$ and $w$ as the radial and axial velocity components, respectively. The geometry of the stenosis is defined as Mekheimer and El Kot [25]

$$
\begin{aligned}
& h(z)=d(z)\left[1-\eta\left(b^{n-1}(z-a)-(z-a)^{n}\right)\right], \quad a \leq z \leq a+b, \\
& h(z)=d(z), \text { otherwise }
\end{aligned}
$$

with $d(x)=d_{0}+\varepsilon z$, where $d(z)$ is the radius of the tapered arterial segment in the stenotic region, $d 0$ is the radius of the non-tapered artery in the non-stenotic region, is the tapering parameter, $b$ is the length of the stenosis, $n(\geq 2)$ is a parameter determining the shape of the constriction profile and referred to as the shape parameter (the symmetric stenosis occurs for $n=2$ ) and a indicates its location (as shown in Fig.1).

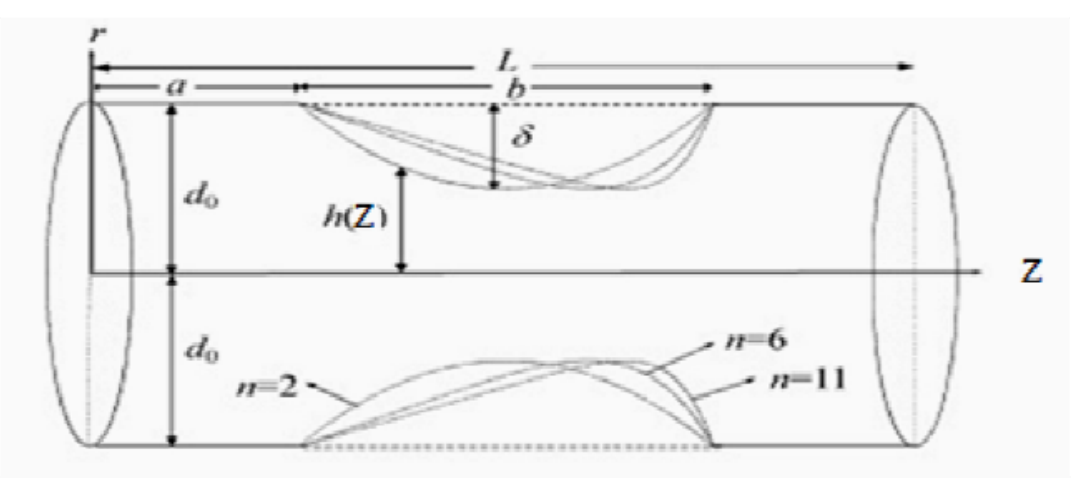

Fig.1. Model for stenosis.

The fluid is assumed to be slightly conducting, so that the magnetic Reynolds number is very small and the induced magnetic field is negligible in comparison with the applied magnetic field. It is also assumed that there is no applied voltage, so that electric field is absent. All the fluid properties are assumed to be constant except that of the influence of the density variation with temperature and concentration in the body force term. A first-order homogeneous chemical reaction is assumed to take place in the flow.

The equations governing the steady incompressible Newtonian fluid with energy and mass concentration equation are given as

$$
\begin{aligned}
& \frac{\partial \bar{u}}{\partial \bar{r}}+\frac{\bar{u}}{\bar{r}}+\frac{\partial \bar{w}}{\partial \bar{z}}=0, \\
& \rho\left[\bar{u} \frac{\partial \bar{u}}{\partial \bar{r}}+\bar{w} \frac{\partial \bar{u}}{\partial \bar{r}}\right]=-\frac{\partial \bar{p}}{\partial \bar{r}}+\frac{\partial}{\partial r}\left[2 \mu \frac{\partial \bar{u}}{\partial \bar{r}}\right]+\frac{2 \mu}{\bar{r}}\left(\frac{\partial \bar{u}}{\partial \bar{r}}-\frac{\bar{u}}{\bar{r}}\right) \frac{\partial}{\partial \bar{z}}\left[\mu\left(\frac{\partial \bar{u}}{\partial \bar{z}}+\frac{\partial \bar{w}}{\partial \bar{r}}\right)\right], \\
& \rho\left[\bar{u} \frac{\partial \bar{w}}{\partial \bar{r}}+\bar{w} \frac{\partial \bar{w}}{\partial \bar{z}}\right]=-\frac{\partial \bar{p}}{\partial \bar{z}}+\frac{\partial}{\partial \bar{z}}\left[2 \mu \frac{\partial \bar{w}}{\partial \bar{z}}\right]+\frac{1}{\bar{r}} \frac{\partial}{\partial \bar{r}}\left[\mu \bar{r}\left(\frac{\partial \bar{u}}{\partial \bar{z}}+\frac{\partial \bar{w}}{\partial \bar{r}}\right)\right]-\sigma_{l} \mu_{o}^{2} H_{0}^{2} \bar{w}-\frac{\mu \bar{w}}{k_{l}},
\end{aligned}
$$




$$
\begin{aligned}
& \rho c_{p}\left[\bar{u} \frac{\partial \bar{T}}{\partial \bar{r}}+\bar{w} \frac{\partial \bar{T}}{\partial \bar{z}}\right]=\frac{k}{\bar{r}} \frac{\partial}{\partial \bar{r}}\left(\bar{r} \frac{\partial \bar{T}}{\partial \bar{r}}\right)+\left(\frac{\partial \bar{w}}{\partial \bar{r}}\right)^{2}-\frac{\partial q_{r}}{\partial \bar{z}}+\frac{D_{m} K_{T}}{C_{m}}\left(\frac{\partial^{2} \bar{C}}{\partial \bar{r}^{2}}+\frac{1}{\bar{r}} \frac{\partial \bar{C}}{\partial \bar{r}}+\frac{\partial^{2} \bar{C}}{\partial \bar{z}^{2}}\right), \\
& \left(\bar{u} \frac{\partial}{\partial \bar{r}}+\bar{w} \frac{\partial}{\partial \bar{z}}\right) \bar{C}=D\left(\frac{\partial^{2} \bar{C}}{\partial \bar{r}^{2}}+\frac{1}{\bar{r}} \frac{\partial \bar{C}}{\partial \bar{r}}+\frac{\partial^{2} \bar{C}}{\partial \bar{z}^{2}}\right)+\frac{D K_{T}}{T_{m}}\left(\frac{\partial^{2} \bar{T}}{\partial \bar{r}^{2}}+\frac{1}{\bar{r}} \frac{\partial \bar{T}}{\partial \bar{r}}+\frac{\partial^{2} \bar{T}}{\partial \bar{z}^{2}}\right)-k \bar{C} .
\end{aligned}
$$

Corresponding boundary conditions are given by

$$
\begin{aligned}
& \frac{\partial \bar{w}}{\partial \bar{r}}=0, \quad \frac{\partial \bar{T}}{\partial r} 0, \quad \frac{\partial \bar{C}}{\partial \bar{r}}=0 \quad \text { at } \quad r=0, \\
& \bar{w}=\bar{T}=\bar{C}=0 \quad \text { at } \quad r=h(z)
\end{aligned}
$$

where $\quad h(z)=d(z)\left[1-\eta\left(b^{n-1}(z-a)-(z-a)^{n}\right)\right]$

Introducing following dimensionless parameters

$$
\begin{aligned}
& r=\frac{r}{d_{0}}, \quad z=\frac{\bar{z}}{b}, \quad w=\frac{\bar{w}}{u_{0}}, \quad u=\frac{b \bar{u}}{u_{0} \delta}, \quad \mathrm{Re}=\frac{\rho b u_{0}}{\mu}, \quad M=\sigma_{1} \mu_{m} H_{0} \sqrt{\frac{\sigma_{1}}{\mu}}, \\
& K=\frac{k_{1}}{d_{0}}, \quad \mathrm{Ec}=\frac{u_{0}^{2}}{c_{p}\left(T_{0}-T_{1}\right)}, \quad \operatorname{Pr}=\frac{\mu c_{p}}{k}, \quad R=\frac{k^{*} k_{\infty}}{4 \sigma^{*} T^{3}}, \quad \mathrm{Sc}=\frac{\mu}{\rho D_{m}}, \\
& \mathrm{Sr}=\frac{D_{m} K_{T} \rho\left(T_{0}-T_{1}\right)}{\mu T_{m}\left(C_{0}-C_{1}\right)}, \quad D_{f}=\frac{D_{m K_{T}\left(C_{0}-C_{l}\right)}}{C_{s} c_{p}\left(T_{0}-T_{1}\right)}, \quad K_{r}=\frac{k_{1} \bar{u}}{u_{0}^{2}},
\end{aligned}
$$

and by adopting additional conditions (Mekheimer and E1 Kot [25])

$$
\begin{aligned}
& \operatorname{Re} \frac{\delta n^{\frac{1}{n-1}}}{b} \ll 1, \\
& \frac{d_{0} n^{\frac{1}{n-1}}}{b} \sim O(1),
\end{aligned}
$$

the dimensionless equations take the following form for the case of mild stenosis $\left(\frac{\delta}{d_{0}} \ll 1\right)$

$$
\begin{aligned}
& \frac{\partial u}{\partial r}+\frac{u}{r}+\frac{\partial w}{\partial z}=0, \\
& \frac{\partial p}{\partial r}=0
\end{aligned}
$$




$$
\begin{aligned}
& \frac{\partial p}{\partial z}=\frac{1}{r} \frac{\partial}{\partial r}\left[r\left(\frac{\partial w}{\partial r}\right)\right]-\left(M^{2}+\frac{1}{K}\right) w \\
& 0=\left(1+\frac{4}{3 R}\right) \frac{1}{r} \frac{\partial}{\partial r}\left[r\left(\frac{\partial \theta}{\partial r}\right)\right]+\operatorname{Ec} \operatorname{Pr}\left(\left(\frac{\partial w}{\partial r}\right)^{2}\right)+D_{f}\left(\frac{1}{r}\left(\frac{\partial}{\partial r}\left(r \frac{\partial \sigma}{\partial r}\right)\right)\right), \\
& \frac{1}{\operatorname{Sc}}\left(\frac{1}{r} \frac{\partial}{\partial r}\left(r \frac{\partial \sigma}{\partial r}\right)\right)+\operatorname{Sr}\left(\frac{1}{r} \frac{\partial}{\partial r}\left(r \frac{\partial \theta}{\partial r}\right)\right)=0,
\end{aligned}
$$

with boundary conditions

$$
\begin{aligned}
& \frac{\partial w}{\partial r}=0, \quad \frac{\partial \theta}{\partial r}=0, \quad \frac{\partial \sigma}{\partial r}=0 \quad \text { at } \quad r=0, \\
& w=0, \quad \theta=0, \quad \sigma=0 \quad \text { at } \quad r=h(z),
\end{aligned}
$$

where

$$
h(z)=(1+\varepsilon z)\left[1-\eta_{l}\left((z-\vartheta)-(z-\vartheta)^{n}\right)\right] \text {. }
$$

\section{Solution of the problem}

The finite difference method is applied to solve dimensionless equations. First order derivatives are discretized by the forward difference method and second order derivatives are discretized by the central difference method. Variation in the radial direction is taken by varying $i$ and in the axial direction by varying $j$. Step size is chosen as 0.001 . The discretized equations obtained are as follows

$$
\begin{aligned}
& \left(\frac{\partial p}{\partial z}\right)_{i, j}=\frac{1}{r_{i, j}} \frac{w_{i+1, j}-w_{i, j}}{\Delta r}+\frac{w_{i+1, j}-2 w_{i, j}+w_{i-1, j}}{(\Delta r)^{2}}-\left(M^{2}+\frac{1}{K}\right) w_{i, j}, \\
& \left.0=\left(1+\frac{4}{3 R}\right) \frac{1}{r_{i, j}} \frac{\theta_{i+1, j}-\theta_{i, j}}{\Delta r}+\frac{\theta_{i+1, j}-2 \theta_{i, j}+\theta_{i-1, j}}{(\Delta r)^{2}}\right)+\operatorname{Ec} \operatorname{Pr}\left(\left(\frac{w_{i+1, j}-w_{i, j}}{\Delta r}\right)^{2}\right)+ \\
& +D_{f}\left(\frac{1}{r_{i, j}} \frac{\sigma_{i+1, j}-\sigma_{i, j}}{\Delta r}+\frac{\sigma_{i+1, j}-2 \sigma_{i, j}+\sigma_{i-l, j}}{(\Delta r)^{2}}\right) \frac{1}{\operatorname{Sc}}\left(\frac{1}{r_{i, j}} \frac{\sigma_{i+1, j}-\sigma_{i, j}}{\Delta r}+\frac{\sigma_{i+1, j}-2 \sigma_{i, j}+\sigma_{i-l, j}}{(\Delta r)^{2}}\right)+ \\
& +\operatorname{Sr}\left(\frac{1}{r_{i, j}} \frac{\theta_{i+1, j}-\theta_{i, j}}{\Delta r}+\frac{\theta_{i+1, j}-2 \theta_{i, j}+\theta_{i-1, j}}{(\Delta r)^{2}}\right)-K_{r} \sigma_{i} .
\end{aligned}
$$

These discretized equations are converted into algebraic equations by taking varying $i$ from 1 to $h(z)$ and $j$ is varied from 0 to 1 . Algebraic equations form a tridiagonal matrix at every $j$ step and are solved by Thomas Algorithm in MATLAB.

\section{Results and discussion}

Blood flow characteristics are computed for a specific set of values of the different parameters involved in the model analysis and are presented graphically. Various effects on Newtonian incompressible 
blood flow in the presence of a magnetic field in a porous diverging tapered artery are observed. In order to get a physical insight, numerical calculations for the axial velocity, temperature, concentration for various parameters have been carried out. Values chosen for numerical solutions are $z=0.5, n=2$, $\vartheta=0, \varepsilon=0.005, \delta^{*}=0.6$ and other dimensionless parameters are varied to understand blood flow behavior. In order to verify the accuracy of the present method, we have compared our results with earlier reported results for velocity considering magnetic and porous effects to be zero. The comparisons are found to be in good agreement, as shown in Tab.1.

Table 1. Comparison of results.

\begin{tabular}{|l|l|l|}
\hline \multicolumn{1}{|c|}{$r$} & $w$ (Present results) & $w$ (Naddem et al. [26]) \\
\hline-1.0 & 0.000 & 0.000 \\
\hline-0.8 & 0.302 & 0.303 \\
\hline-0.6 & 0.538 & 0.535 \\
\hline-0.4 & 0.700 & 0.703 \\
\hline-0.2 & 0.809 & 0.802 \\
\hline 0.2 & 0.809 & 0.802 \\
\hline 0.4 & 0.700 & 0.703 \\
\hline 0.6 & 0.538 & 0.535 \\
\hline 0.8 & 0.302 & 0.303 \\
\hline 1.0 & 0.000 & 0.000 \\
\hline
\end{tabular}

\section{Velocity profiles}

The velocity profile for different values of $M, K$ and $\varepsilon$ are shown in Figs 2 to 4 . Velocity is found to decrease with an increase in the magnetic field, shown in Fig.2, as the magnetic field introduces Lorentz force in the electrically conducting fluid. This force acts against blood flow which reduces velocity with an increase in the magnetic field. These results qualitatively agree with the expectations, since the magnetic field exerts a retarding force on the natural convection flow. It is also observed that velocity increases with an increase in $K$ as shown in Fig.3. Physically, this means that the porous medium impact on the boundary layer growth is significant due to the increase in the thickness of the thermal boundary layer. As expected, an increase in the permeability of the porous medium leads to the rise in the flow of fluid through it. When the holes of the porous medium become large, the resistance of the medium may be neglected. Variation due to a change in the tapering angle is shown in Fig.4, and it is seen that converging artery has higher axial velocity as compared to non-tapered and diverging artery. 


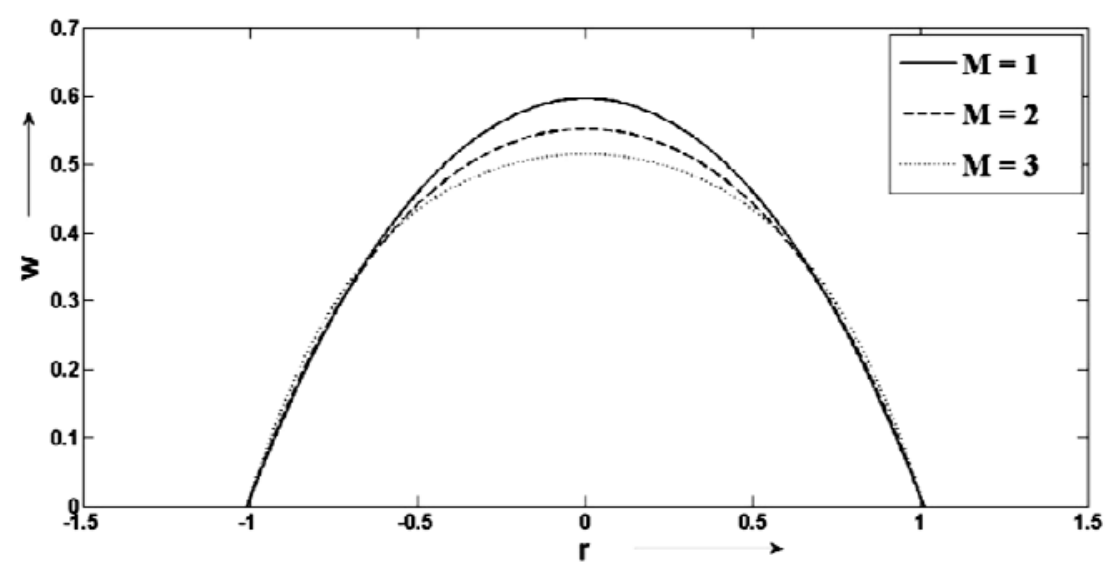

Fig.2. Velocity profile for varying $M$.

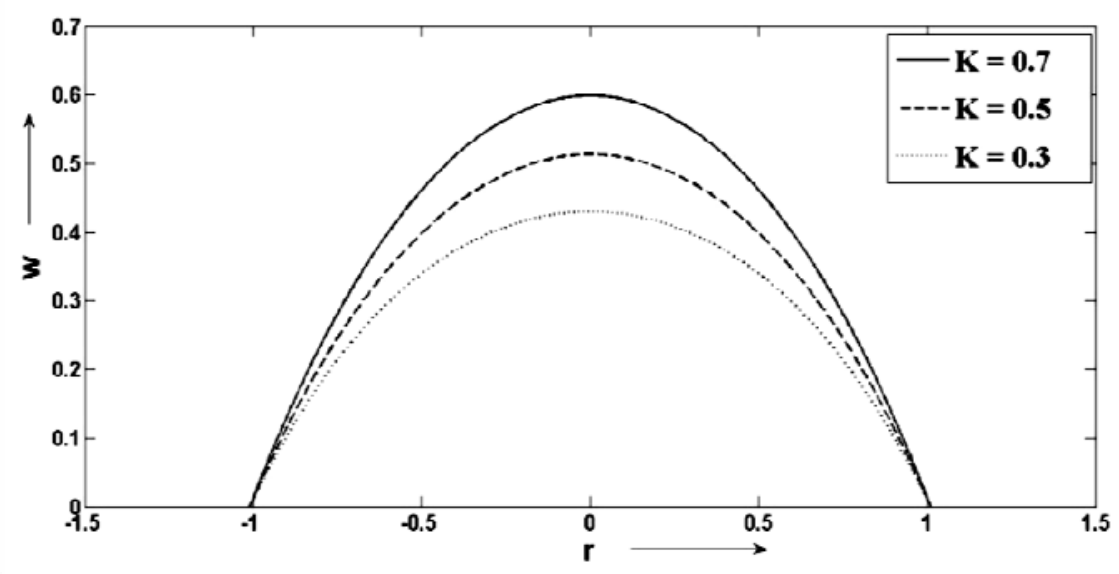

Fig.3. Velocity profile for varying $K$.

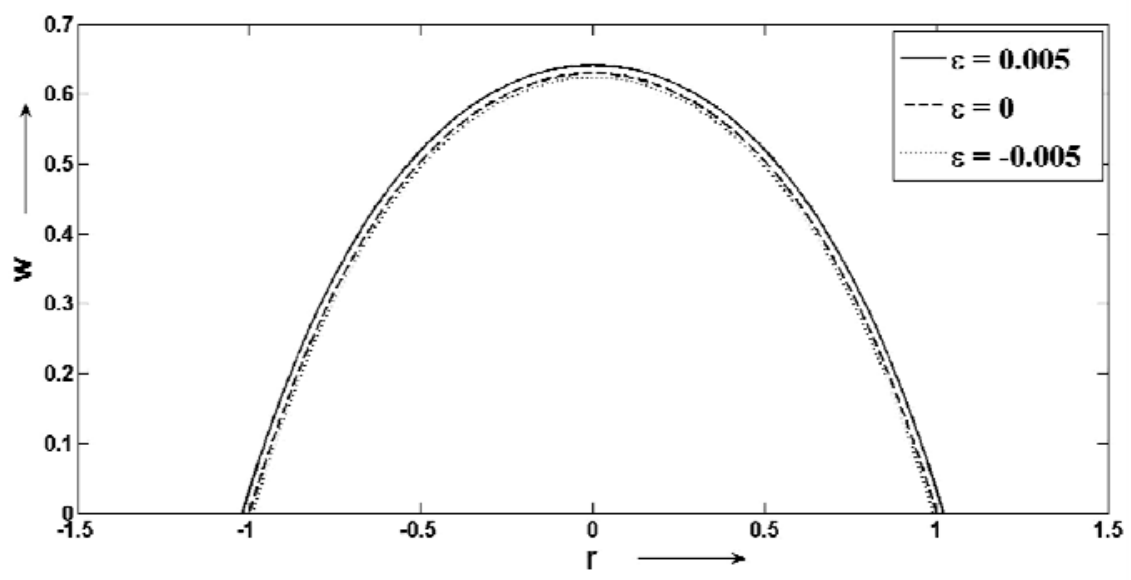

Fig.4. Velocity profile for varying $\varepsilon$. 


\section{Temperature profiles}

Temperature profiles for different values of $M, R, \operatorname{Pr}, K, \mathrm{Df}, \mathrm{Br}(=\mathrm{Ec} * \mathrm{Pr}), \mathrm{Sr}$ are shown in Figs 5 to 10. Figure 5 shows the change in temperature when $\mathrm{Br}$ is varied. It is found that an increase in $\mathrm{Br}$ value increases temperature which also means there is a increase in temperature with an increase in the Eckert number as $\mathrm{Br}=\mathrm{Ec} * \mathrm{Pr}$. Figure 6 shows the variation of temperature with $K$ and it is found that as $K$ increases the temperature of blood increases. It is observed from Fig. 8 that the temperature of blood in the presence of stenosis decreases with an increase in the magnetic field. It is also observed that temperature increases with an increase in the radiation parameter $R$ as shown in Fig.9. Radiation acts as a heat source within the blood, the arterial blood temperature (and hence also that along the centerline) should gradually increase with increasing radiation dosage. Variation of temperature with the Dufour number is shown in Fig.10. It is found that an increase in the Dufour effect increases temperature. Figure 11 shows variation of temperature with Sr. Changes are very small and temperature increases with an increase in Sr.

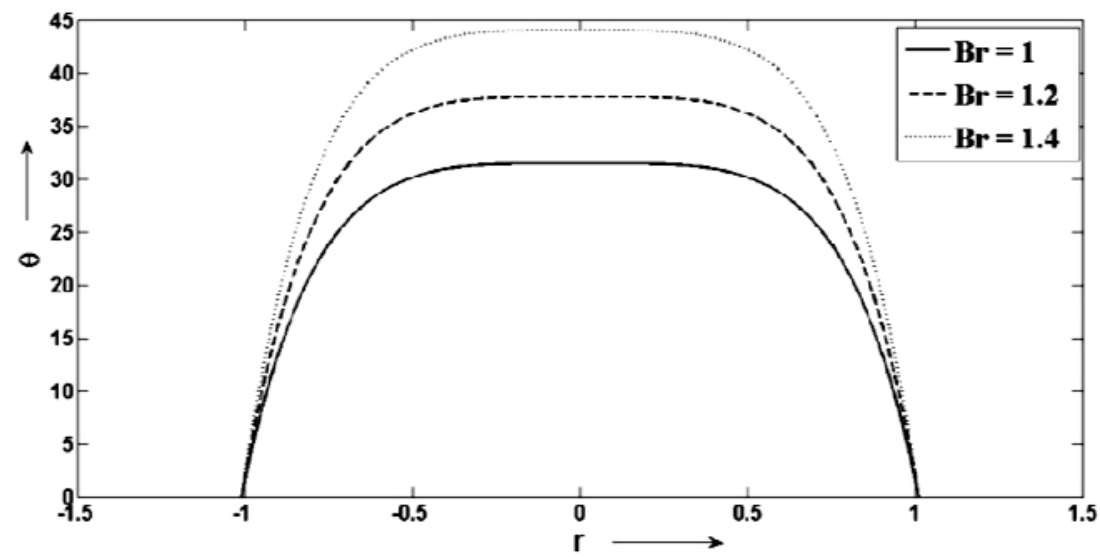

Fig.5. Temperature profile for varying $\mathrm{Br}$.

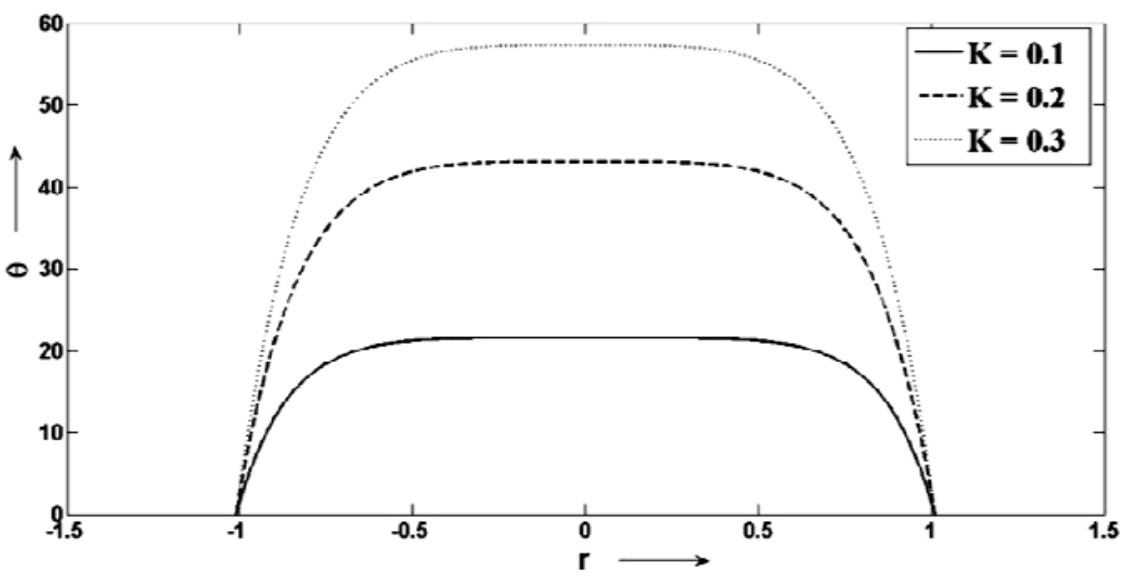

Fig.6. Temperature profile for varying $K$. 


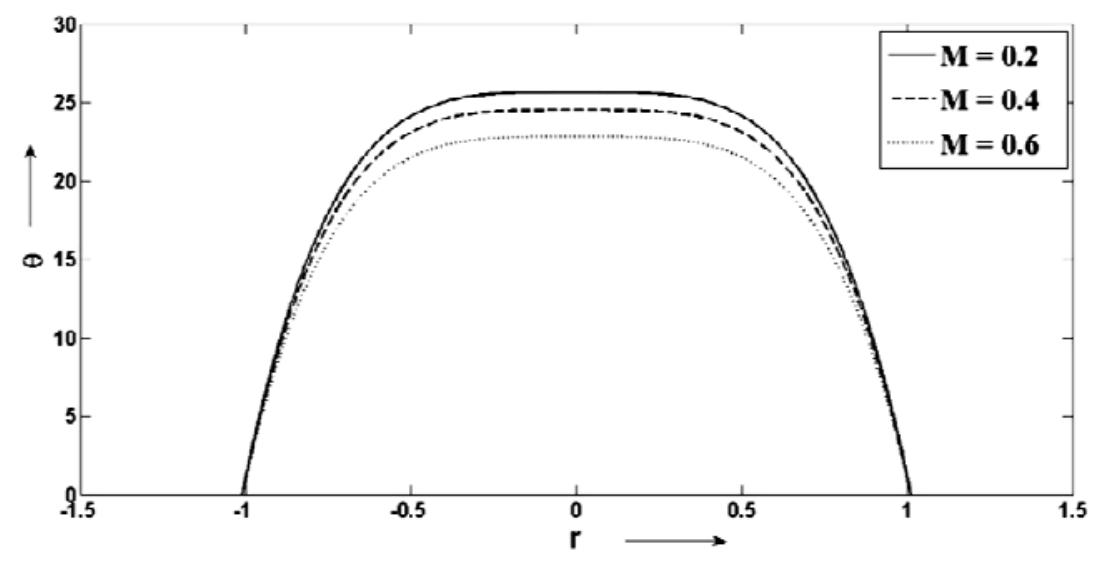

Fig.7. Temperature profile for varying $M$.

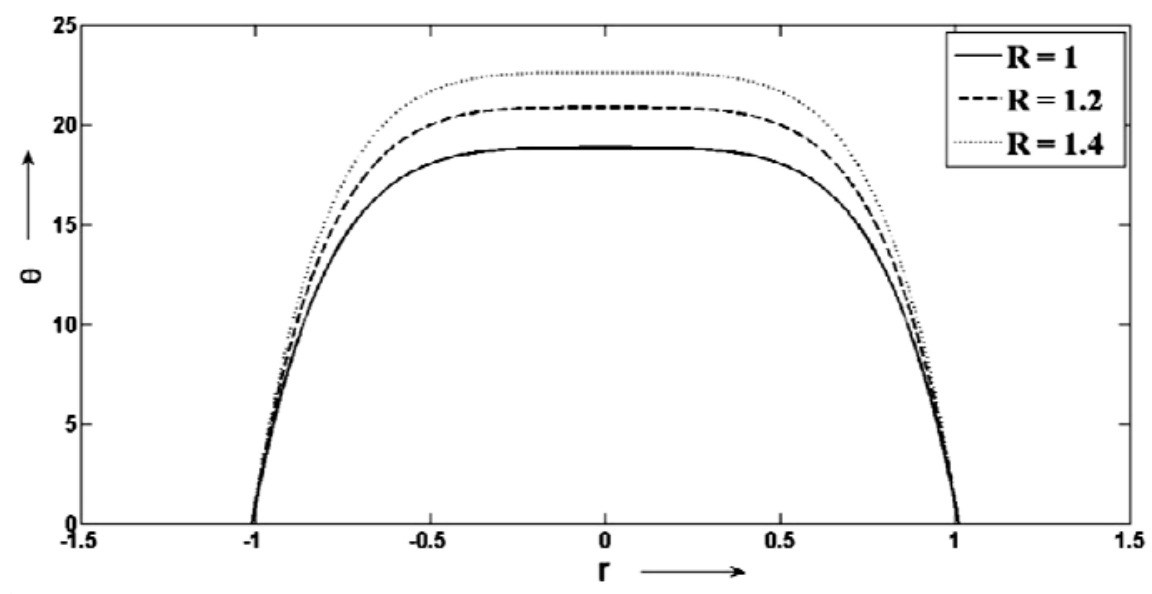

Fig.8. Temperature profile for varying $R$.

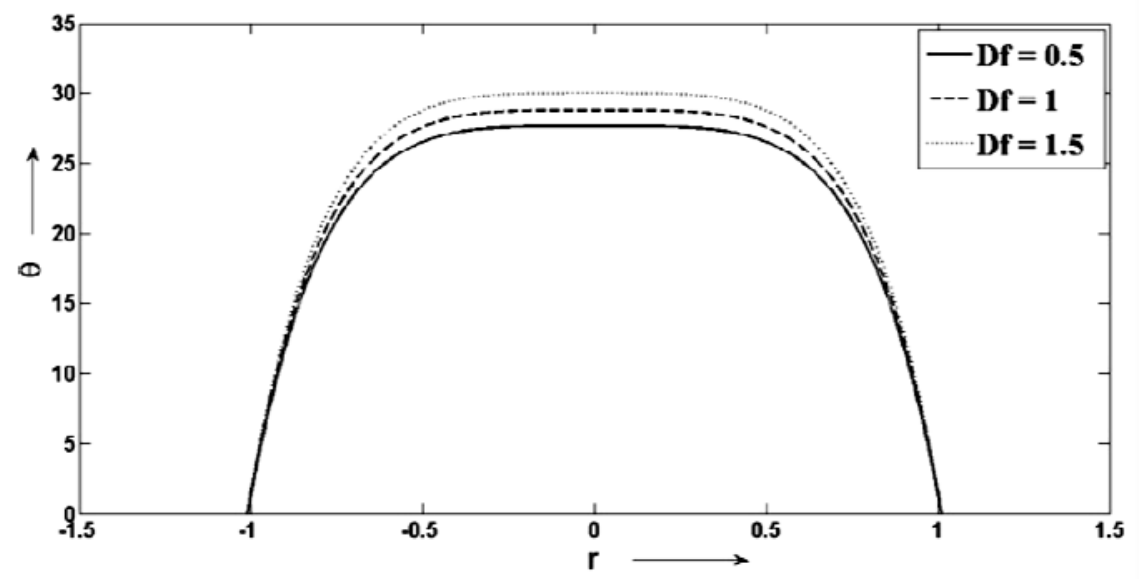

Fig.9. Temperature profile for varying Df. 


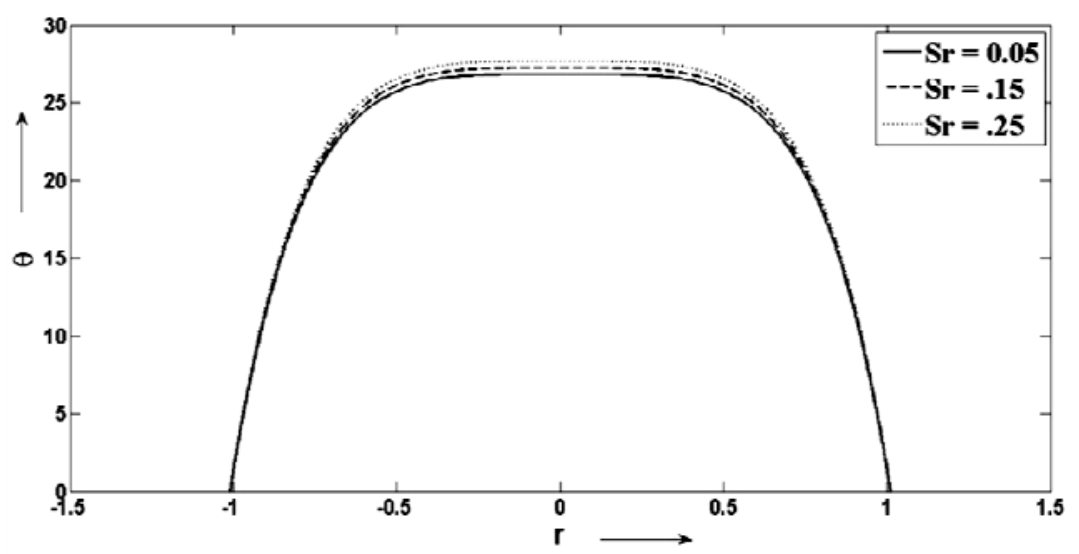

Fig.10. Temperature profile for varying Sr.

\section{Concentration profiles}

Concentration profiles for different values of $\mathrm{Sc}, \mathrm{Sr}, \mathrm{Br}, M$ and $K r$ are shown in Figs 11-15. It is observed that with an increase in the Brickmann number Br, Schmidt number Sc and Soret number Sr the concentration profile decreases, while it increases with an increase in the magnetic field $M$ and chemical reaction parameter $K r$. As the Schmidt number increases, the concentration decreases. This causes the concentration buoyancy effects to decrease yielding a reduction in the fluid velocity.

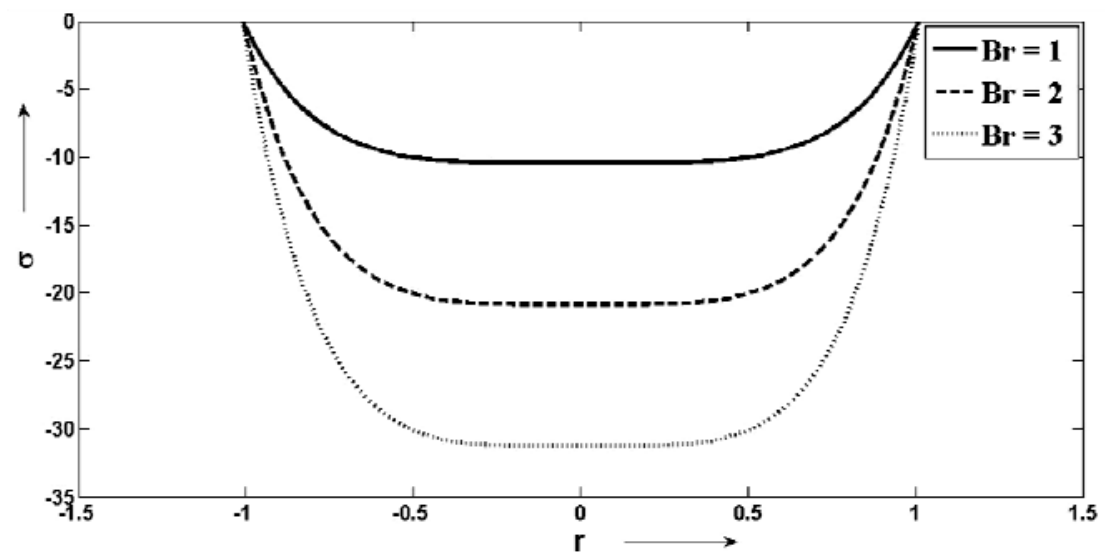

Fig.11. Concentration profile for varying $\mathrm{Br}$.

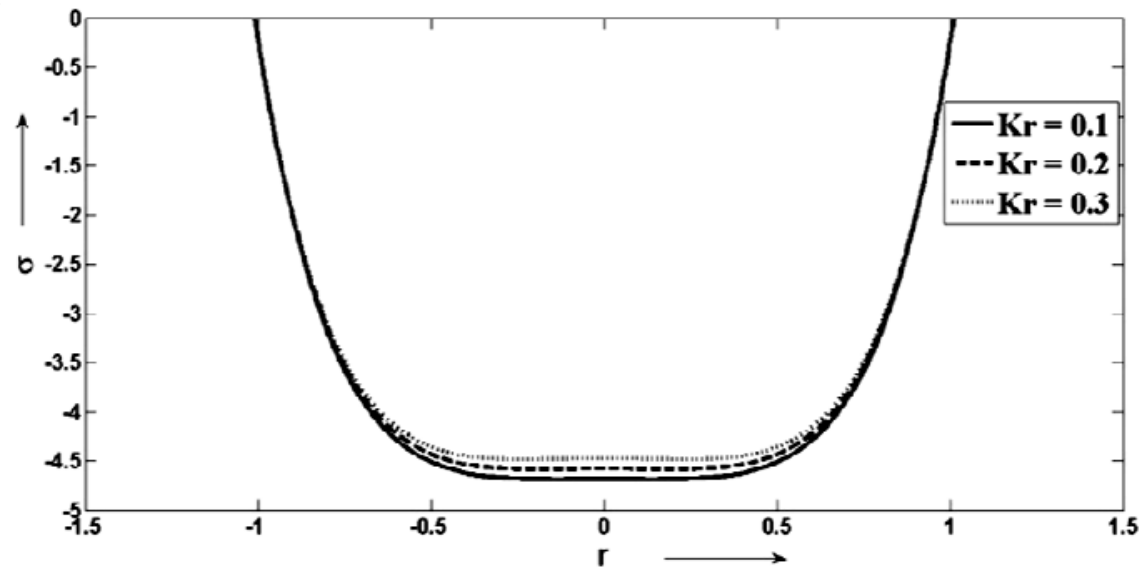

Fig.12. Concentration profile for varying $K r$. 


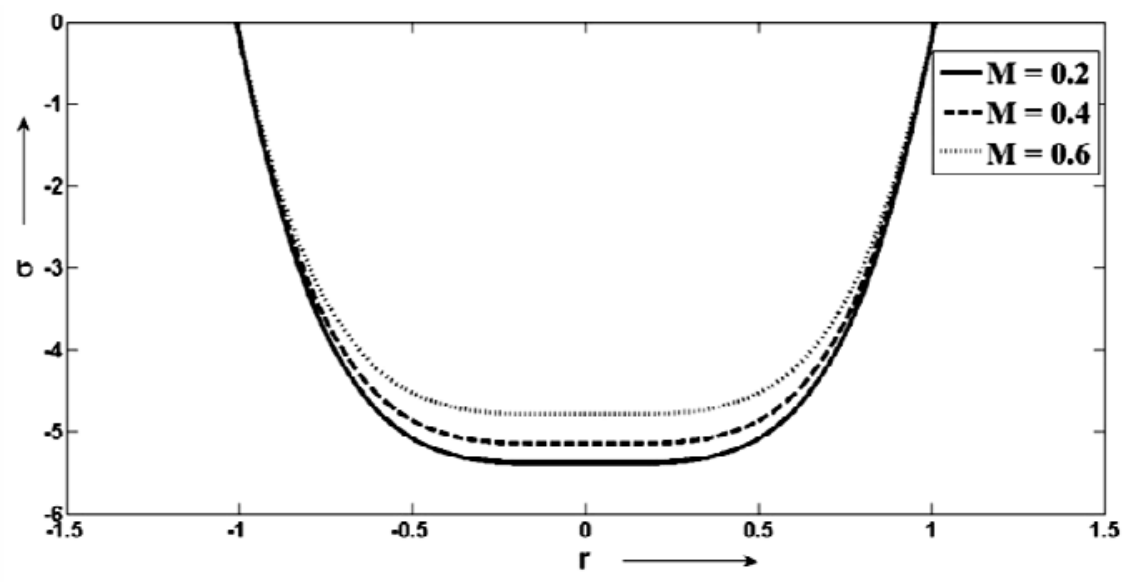

Fig.13. Concentration profile for varying $M$.

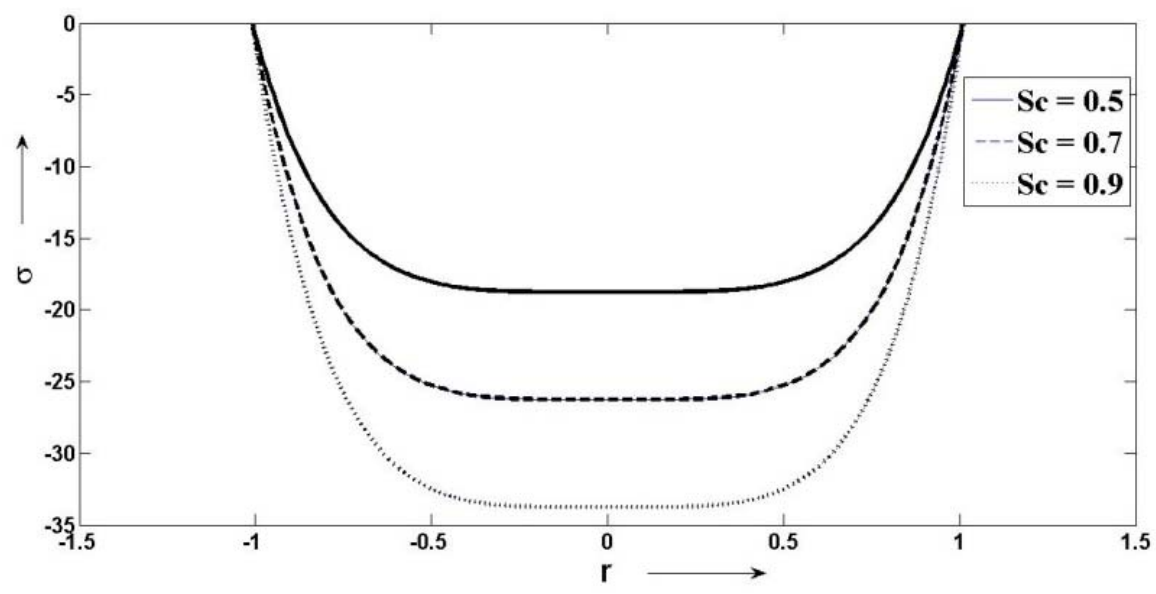

Fig.14. Concentration profile for varying Sc.

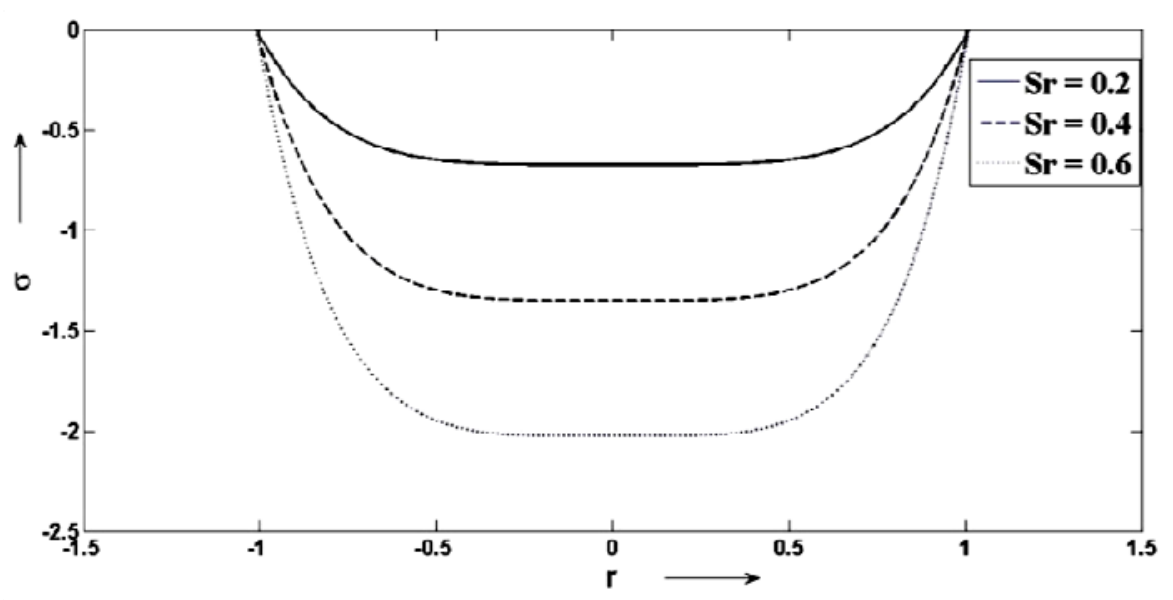

Fig.15. Concentration profile for varying $\mathrm{Sr}$.

\section{Conclusions}

MHD blood flow through a stenosed artery with Soret and Dufour effects in the presence of thermal radiation has been studied. The resulting equations were solved numerically by the finite difference 
scheme. In order to check the accuracy and validate the present method, results are compared with the existing results. The effects of the thermal radiation, the magnetic field, Soret and Dufour parameters on the stenosis geometry have been observed throughout and the results obtained are of physiological interest and clinical applications. The study reveals that for patients undergoing thermal radiation therapy, the resistance to blood flow due to a magnetic field and stenosis is reduced by increasing the thermal radiation absorption.

\section{Nomenclature}

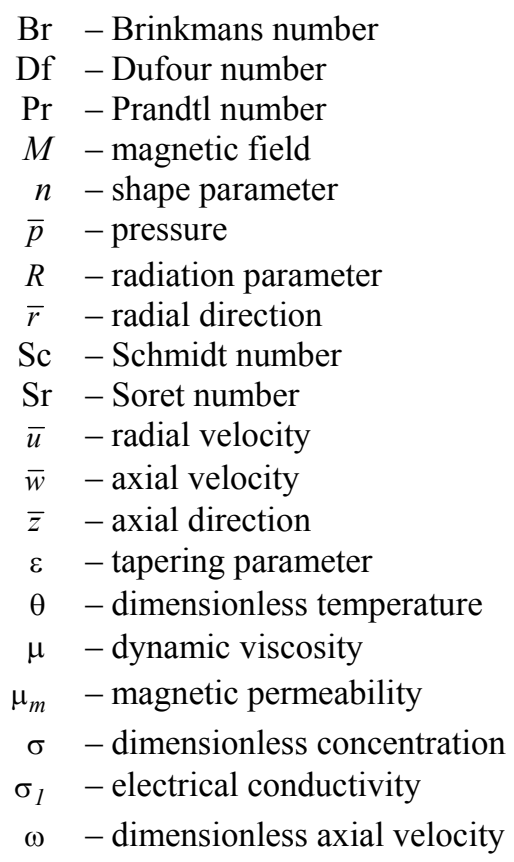

\section{References}

[1] Higashi T., Yamagishi A., Takeuchi T., Kawaguchi N., Sagawa S., Onishi S. and Date M. (1993): Orientation of erythrocytes in a strong static magnetic field. - Blood, vol.82, pp.1328-1334.

[2] Panja S. and Sengupta P.R. (1996): Hydromagnetic flow of Reiner Rivlin fluid between two coaxial circular cylinders with porous walls. - Comput. Math. Appl., vol.32, pp.1-43.

[3] Shukla J.B., Parihar R.S. and Gupta S.P. (1980): Biorheological aspects of blood flow through artery with mild stenosis: effects of peripheral layer. - Biorheology, vol.17, pp.403-410.

[4] Chakravarty S. and Sannigrahi A. (1999): A nonlinear mathematical model of blood flow in a constricted artery experiencing body acceleration. - Math. Comput. Model, vol.29, pp.9-25.

[5] Haik Y., Pai V. and Chen C.-J. (2001): Apparent viscosity of human blood in a high static magnetic field. J. Magn. Magn. Mater., vol.225, No.1-2, pp.180-186.

[6] Yadav R.P., Harminder S. and Bhoopal S. (2008): Experimental studies on blood flow in stenosis arteries in presence of magnetic field. - Ultra Sci., vol.20, No.3, pp.499-504.

[7] Sharma B.K., Mishra A. and Gupta S. (2013): Heat and mass transfer in magneto-biofluid flow through a nonDarcian porous medium with Joule effect. - J. Eng. Phys. and Thermo Phy., vol.86, No.4, pp.716-725.

[8] Sinha A., Mishra J.C. and Shit G.C. (2016): Effect of heat transfer on unsteady MHD flow of blood in a permeable vessel in the presence of non-uniform heat source. - Alexandria Engineering Journal, vol.55, No.3, pp.2023-2033.

[9] Shit G.C. and Roy M. (2016): Effect of induced magnetic field on blood flow through a constricted channel. An analytical approach. - Journal of Mechanics in Medicine and Biology, vol.16, No.03, pp.1650030. 
[10] Rahbari A., Fakour M., Hamzehnezhad A., Vakilabadi M.A. and Ganji D.D. (2017): Heat transfer and fluid flow of blood with nanoparticles through porous vessels in a magnetic field: A quasi-one dimensional analytical approach. - Mathematical Biosciences, vol.283, pp.38-47.

[11] Levin W., Sherar M.D., Cooper B., Hill R.P., Hunt J.W. and Liu F.-F. (1994): Effect of vascular occlusion on tumour temperatures during superficial hyperthermia. - International Journal of Hyperthermia, vol.10, No.4, pp.495-505.

[12] Sharma B.K., Sharma M., Gaur R.K. and Mishra A. (2015): Mathematical modeling of magneto pulsatile blood flow through a porous medium with a heat source. - International Journal of Applied Mechanics and Engineering, vol.20, No.2, pp.385-396.

[13] Sinha A. and Shit G.C. (2015): Electromagnetohydrodynamic flow of blood and heat transfer in a capillary with thermal radiation. - Journal of Magnetism and Magnetic Materials, vol.378, pp.143-151.

[14] Sharma B.K., Sharma M. and Gaur R.K. (2015): Thermal radiation effect on inclined arterial blood flow through a non-Darcian porous medium with magnetic field. - Proceeding: First Thermal and Fluids Engineering Summer Conference, ASTFE Digital Library, vol.17, pp.2159-2168, DOI: 10.1615/TFESC1.bio.013147.

[15] Tripathi B. and Sharma B.K. (2018): Influence of heat and mass transfer on MHD two-phase blood flow with radiation. - AIP Conference Proceedings, vol.1975, 030009:1-9 (2018); doi: 10.1063/1.5042179.

[16] Gnaneswara Reddy M. (2014): Thermal radiation and chemical reaction effects on MHD mixed convective boundary layer slip flow in a porous medium with heat source and Ohmic heating. - Eur. Phys. J. Plus, 129:41.

[17] Sharma M. and Gaur R.K. (2017): Effect of variable viscosity on chemically reacting magneto-blood flow with heat and mass transfer. - Global Journal of Pure and Applied Mathematics, vol.13, Special Issue No.3, pp.26-35.

[18] Gnaneswara Reddy M. (2017). Velocity and thermal slip effects on MHD third order blood flow in an irregular channel though a porous medium with homogeneous/ heterogeneous reactions. - Nonlinear Engineering, vol..6, No.3, pp.167-177.

[19] Eckert E.R.G. and Drake R.M. (1974): Analysis of Heat and Mass Transfer. - New York, NY, USA: MC Graw-Hill.

[20] Sharma B.K., Yadav K., Mishra N.K. and Chaudhary R.C. (2012): Soret and Dufour effects on unsteady MHD mixed convection flow past a radiative vertical porous plate embedded in a porous medium with chemical reaction. - Applied Mathematics, vol.3, No.7, pp.717-723.

[21] Sharma B.K., Gupta S., Krishna V.V. and Bhargavi R.J. (2014): Soret and Dufour effects on an unsteady MHD mixed convective flow past an infinite vertical plate with Ohmic dissipation and heat source. - Afrika Matematika, vol.25, No.3, pp.799-825.

[22] Hayat T., Shehzad S.A. and Alsaedi A. (2012): Soret and Dufour effects on the magnetohydrodynamic (MHD) flow of the Casson fluid over a stretched surface. - Appl. Math. Mech. -Engl. Ed., vol.33, No.10, pp.1301-1312. DOI 10.1007/s10483-012-1623-6.

[23] Hayat T., Zahir H., Tanveer A. and Alsaedi A. (2017): Soret and Dufour effects on MHD peristaltic transport of Jeffrey fluid in a curved channel with convective boundary conditions. - PLoS ONE, vol.12, No.2, e0164854. https://doi.org/10.1371/ journal.pone. 0164854.

[24] Sharma B.K., Sharma M. and Gaur R.K. (2018): Effect of chemical reaction on Jeffrey fluid model of blood flow through tapered artery with thermo-diffusion and diffuso-thermal gradients. - Proceeding: Third Thermal and Fluids Engineering Conference, ASTFE Digital Library, vol.18, pp.1103-1115, DOI: 10.1615/TFEC2018.bio.022113.

[25] Mekheimer Kh.S. and El Kot M.A. (2008): The micropolar fluid model for blood flow through a tapered artery with a stenosis. - Acta Mech. Sin., vol.24, No.6, pp.637-644.

[26] Nadeem S., Akbar N.S., Hayat T. and Hendi A.A. (2012): Influence of heat and mass transfer on newtonian biomagnetic fluid of blood flow through a tapered porous arteries with a stenosis. - Transp. Porous Med., vol.91, pp.81-100. DOI 10.1007/s11242-011-9834-6.

Received: October 11, 2017

Revised: July 12, 2018 